\title{
¿Indie vs. mainstream? Una aproximación a la relación entre industria musical y radio musical pública en España
}

Lola Costa Gálvez, Universitat Autònoma de Barcelona - doloresgalvez@gmail.com

Resumen

Radio y música han estado ligadas desde sus inicios. Además, en el caso de la radio musical pública ha servido para articular un discurso alrededor de la industria musical y especialmente la de carácter más local. En el presente artículo haremos una aproximación a la relación entre radio musical pública e industria musical. Esta relación ha tenido frecuentemente su traducción en una programación basada en la dicotomía mainstream/indie, la protección de las lenguas cooficiales y la promoción de los artistas emergentes, lo que ha servido para definir y justificar la existencia de algunas emisoras. Concretamente se tomará el caso de estudio de cuatro radios musicales públicas en España, con modelos consolidados centrados en la música popular (Canal Fiesta Radio, Gaztea Irratia iCat.cat y Radio 3). En un contexto de incertidumbre, no solo para la radio sino también para la música, la unión de ambas industrias culturales puede suponer su salvaguarda.

\section{Palabras clave}

Radio musical, servicio público, España, comunidades autónomas, programación, industria musical.

Abstract

Radio and music have been bound from the beginnings. Moreover, public music radio has used the discourse about local music industry as a justification of its existence. In the present article we will approach the relationship between public music radio and music industry. This association has been frequently translated into a programming strategy based in mainstream/indie dichotomy, the protection of coofficial languages and the promotion of under-the-radar musicians, what has been useful for defining and justifying the existence of some stations. In particular, we will focus on the case study of four public music radios in Spain, with consolidated models focused on popular music (Canal Fiesta Radio, Gaztea Irratia iCat.cat, and Radio 3). In an uncertainty context, not only for public service media but also for the music industry, the union of both cultural industries can become their safeguard.

\section{Keywords}

Music radio, public service media, Spain, authonomous communities, programming, music industry.

Sumario

1. Introducción. 2. Metodología. 3. Radio musical pública en España y música popular: cuatro modelos consolidados. 4. Radio musical pública e industria musical: un entendimiento necesario. 5. Conclusiones. 6. Bibliografía. 


\section{Introducción}

La retroalimentación entre radio y música ha sido importante desde sus inicios (Frith, 1987; Percival, 2011) amén de ser una importante fuente de ingresos (IFPI, 2010b) lo que ha situado a la radio musical en una posición hegemónica durante mucho tiempo (Rothenbuhler, 2004). Además, la contribución de la radio pública a la formación de escenas musicales ha sido importante desde sus inicios, recordemos los casos de Radio 3 con la llamada «movida» (Fouce, 2008) o el papel de la BBC Radio 1 con el denominado «britpop» (Gilbert y Pearson, 2003).

En el ámbito radiofónico el servicio público está frecuentemente unido a la información y poca atención se ha prestado al ámbito musical, aunque la apuesta de los servicios públicos europeos por la especialización radiofónica es clara (Bonet et al., 2011; Hendy, 2000a). La dicotomía mainstream/indie ha sido fundamental en la programación radiofónica musical desde sus inicios. Además, en el caso de la radio musical de servicio público debería ir más allá del ratings by day, reputation by night o el heasy/hard listening lo que ha servido para desarrollar y justificar otros aspectos como la protección de las lenguas cooficiales o la promoción de los artistas emergentes. Son precisamente las emisoras musicales las que pueden suponer una salvaguarda para el servicio público y un apoyo fundamental para la industria musical especialmente la de carácter más independiente y local.

En el presente artículo analizaremos estos aspectos tomando como caso de estudio cuatro radios musicales públicas en España centradas en la música popular (Canal Fiesta Radio, Gaztea Irratia iCat.cat y Radio 3) ${ }^{1}$. Concretamente nos planteamos responder de manera exploratoria a la siguiente pregunta:

¿Cómo aborda la radio musical pública en España la relación con la industria musical?

\section{Metodología}

Para la realización de este artículo retomaremos algunos datos de una investigación realizada por la autora². Concretamente se recogerán citas de entrevistas a los directores de antena y jefes de programación de las citadas emisoras para contextualizar la relación con la industria musical. La relación de entrevistados es la siguiente: Araceli Limón (Canal Fiesta Radio), Jon Lamarca (Gaztea Irratia), Carles Aledo y Lucía Flores (iCat.cat) y Tomás Fernando Flores (Radio 3). De este modo y siguiendo la clasificación de Martí (1990) tenemos la siguiente muestra:

Tabla 1. Clasificación de la muestra de radio musical pública en España según tipología (programática) (2016).

\begin{tabular}{|c|c|c|c|}
\hline Tipología (programática) & Corporación audiovisual & Radio & \\
\hline \multirow[t]{11}{*}{ ESPECIALIZADA } & \multirow{9}{*}{ RADIOFÓRMULA MUSICAL } & \multirow{6}{*}{$\begin{array}{l}\text { CCMA } \\
\text { (Corporació Catalana de Mitjans Audiovisuals) }\end{array}$} & iCatJazz \\
\hline & & & iCatMón \\
\hline & & & iCatRumba \\
\hline & & & iCatrònica \\
\hline & & & TotCat \\
\hline & & & \\
\hline & & $\begin{array}{l}\text { EITB } \\
\text { (Euskal Irrati Telebista) }\end{array}$ & Gaztea Irratia \\
\hline & & RTVA & \\
\hline & & (Radio y Televisión de Andalucía) & Canal Fiesta Radio \\
\hline & \multirow[t]{2}{*}{ TEMÁTICA MUSICAL } & $\begin{array}{l}\text { CCMA } \\
\text { (Corporació Catalana de Mitjans Audiovisuals) }\end{array}$ & iCat.cat ${ }^{4}$ \\
\hline & & $\begin{array}{l}\text { RTVE } \\
\text { (Radiotelevisión Española) }\end{array}$ & Radio 3 \\
\hline
\end{tabular}

Fuente: elaboración propia a partir de los datos de las páginas web de CCMA, EITB, RTVA y RTVE.

\footnotetext{
${ }^{1}$ Aunque es cierto que existen otras emisoras públicas centradas en la música popular como OR Música en la Región de Murcia o Son Galicia Radio y RG Música en Galicia, los graves recortes tanto de personal como presupuestarios las han conducido a una situación de falta de interés por parte de las propias corporaciones.

2 Concretamente una tesis doctoral titulada (título, universidad, año) y premiada con (nombre del premio).

${ }^{3}$ Aunque la CCMA hasta 2007 se llamó CCRTV (Corporació Catalana de Radio i Televisió), para evitar confusiones en el siguiente trabajo se mantendrá el nombre actual.
}

${ }^{4}$ Tras el cierre en octubre de 2012 de sus frecuencias en fm, iCat fm, la emisora ha cambiado su nombre a iCat.cat. 


\section{Radio musical pública en España y música popular: cuatro modelos consolidados}

Desde sus inicios la música ha estado ligada a la especialización radiofónica. En España, con la transición y el desarrollo de las corporaciones audiovisuales autonómicas, cuando la radio musical pública toma impulso en España. Es este un ámbito poco estudiado ya que tradicionalmente se ha relacionado a la radio de servicio público con la información y esto queda patente en la mayoría de la producción académica, si bien es cierto que música y radio han sido aspectos ampliamente estudiados (Hennion y Meadel, 1986; Barnard, 1989; Moreno, 1999; Hendy, 2000a; Pedrero Esteban 2000; Crisell, 2006; Amoedo et al., 2008; Gutièrrez et al., 2011; Gallego, 2015). Sin embargo, son pocas las excepciones que han abordado expresamente la tríada radio, música y servicio público (Hendy, 2000b; Long, 2006; Wall y Dubber, 2009; Bonet et al., 2011; autor, año). Idéntica situación encontramos si ponemos el foco en las políticas de comunicación, en las que se ha seguido la estrategia laissez faire, laissez passer respecto a la radio musical (autor, año).

Canal Fiesta Radio (Radio y Televisión de Andalucía, RTVA)

Canal Fiesta Radio surgió en 2001 de la antigua Fórmula 1. Conocido cariñosamente como «El Fiesta», la emisora tuvo unos inicios un tanto truculentos relacionados con la muerte a causa de las drogas de dos jóvenes durante una fiesta del programa Mundo Evassion, que vinieron a ser un epílogo de las desafortunadas palabras en una entrevista de su entonces director, Carmelo Villar, en las que aludía a la tremenda aportación de este programa a la «gran cultura del botellón» (Arboledas citando a Díaz Nosty, 2009).

La idea de una «identidad andaluza» ronda las primeras definiciones de la emisora. Recordemos que en su eslogan se autodenomina «la radio musical de Andalucía», e, incluso, se planteó con la idea de ampliar fronteras y como anécdota tenemos la celebración de su tercer aniversario con «un concierto multitudinario en el Palau Olimpic de Badalona en homenaje a la comunidad andaluza de Cataluña» (RTVA, 2005: 93). Si concretamos, tenemos una emisora «dirigida a la audiencia joven» (Consejería de la Presidencia e Igualdad, 2012: 205), con una clara apuesta por la música en español y «con especial atención a las producciones andaluzas» (Parlamento de Andalucía, RTVA, 2010: 60). Esta clara vocación comercial se puede resumir en la siguiente frase: «Canal Fiesta Radio constituye una emisora musical competitiva con vocación mayoritaria, que no renuncia al espíritu de servicio público de la cadena Canal Sur Radio» (RTVA, 2005: 132)

A nivel programático, el último contrato-programa (Consejería de la Presidencia e Igualdad, 2012) reserva para Canal Fiesta Radio un $75 \%$ de los programas sobre «divulgación de las realizaciones de creadores y artistas andaluces y españoles de la música popular» y un «25\% en espacios de entretenimiento» (Consejería de la Presidencia e Igualdad, 2012: 204). Se ha mantenido un discurso estable y en consonancia con lo que efectivamente ofrece su programación: música pop, comercial y dirigida a un público joven. Además, se hace hincapié en la «identidad» de lo «andaluz», con especial atención a las producciones andaluzas» (Parlamento de Andalucía, RTVA, 2010: 60) y a través de la promoción de la industria cultural musical andaluza, que no de la escena musical andaluza (Consejería de la Presidencia e Igualdad, 2012; RTVA, 2005, 2006).

Gaztea Irratia (Euskal Irrati Telebista, EITB)

La Corporación vasca cuenta con dos emisoras musicales, EITB Musika, definida como una radio «con contenidos específicos de cultura y música de calidad» (EITB, 2011: 5), y Gaztea Irratia, descrita profusamente como una «radio fórmula juvenil» (Departamento de Presidencia, Consejería de Hacienda y Administración Pública, Consejería de Cultura, EITB, 2007; Consejería de Cultura, EITB, 2012; EITB, 2013a).

Tras su cambio de registro a partir de 2005 Gaztea Irratia es descrita como una emisora «juvenil«, con una función de servicio público centrada en la promoción del idioma (Departamento de Presidencia, Consejería de Hacienda y Administración Pública, Consejería de Cultura, EITB, 2007). Así, la cultura y el entretenimiento en su versión más puramente juvenil son respectivamente la emisora, con el euskera como lengua vehicular.

iCat.cat (Corporació Catalana de Mitjans Audiovisuals, CCMA)

La corporación catalana cuenta con una radio y seis bitcasters dedicadas a la música popular. Una de las puntas de lanza de la corporación es iCat.cat definida como un gran contenedor cultural (Bonet et al., 2011; CCRTV, 2007). A esta voluntad cultural debe unirse su consideración como «la radio multimedia del grupo» (CCMA, 2009: 19), llegando a estar presente en plataformas «innovadoras» en su momento como la consola Wii y en la actualidad y tras el apagón de la FM en 2012 generó en una APP propia. Su nacimiento vino aparejado de un proceso denominado «icatización» (CCMA, 2010a: 298), que generó la aparición de canales temáticos en Internet (bitcasters) dedicados a diversos géneros y estilos como el jazz, las músicas del mundo, la música en catalán, la electrónica o la rumba (iCatJazz, iCatMón, iCatRumba, iCatrònica y TotCat).

Como el resto de emisoras de la corporación emite íntegramente en catalán y antes de perder la FM tenía la obligación en virtud de la Ley de Política Lingüística de emitir un $25 \%$ de la música en catalán (Parlament de Catalunya, 1998). En el aspecto musical se destaca el carácter «no comercial» (CCMA, 2011b: 2) y «emergente» (CCMA, 2014b: 14) de promoción de la «escena local catalana» (CCMA, 2011a, 2011b, 2013a, 2014b).

Radio 3 (Radiotelevisión Española, RTVE)

Nacida en 1979 al amparo del Tercer Programa, Radio 3, acuñada «Radio 3 Pop» en sus inicios, comenzó a emitirse como un espacio nocturno «con una duración de cinco horas diarias y centrado en el pop, el rock, el jazz, el folk y la música de vanguardia» (Pedrero Esteban, 2000: 97) y pronto tomó una gran relevancia social de la mano de la llamada «Movida» (Fouce, 2008). Si existe una emisora particularmente difícil de definir en el panorama radiofónico español esa es Radio 3. Así, encontramos calificaciones tan variopintas como emisora de «Música Urbana» (Martí, 1990: 139), «radiofórmula de música joven» (Prado, 1995: 236) o en palabras de la entonces directora de RTVE, Carmen Caffarel: «radio juvenil, cultural e innovadora» (Congreso de los Diputados, 2005: 33).

«Alternatividad» y «no intencionalidad comercial» son términos muy empleados cuando se habla de Radio 3 y precisamente eran los que recogía el Consejo para la Reforma de los Medios de Comunicación de Titularidad del Estado (2005: 96), que consideraba que la emisora «debe abrirse más a la innovación en conexión con nuestra industria discográfica independiente de repertorio nacional», amén de ser una «plataforma de los gustos jóvenes» (ídem). El carácter de contenedor cultural se resaltaba por la entonces directora de RTVE tenía claro lo que abarcan las músicas no comerciales: «Radio 3 aborda músicas no comerciales, desde el folclore tradicional español hasta el rock o las músicas ancestrales de los más apartados rincones del planeta» (Caffarel, 2007: 129). En cuanto al género musical se recalca el término «independiente» e incluso se llega a 
emplear la palabra «indie» (RTVE, 2009: 109, 447), en contraposición con las «listas de éxitos« y las «radios comerciales» (RTVE, 2010: 203), que se suele anteponer a la «calidad»: «la mejor música no comercial» (RTVE, 2013: 341).

\section{Radio musical pública e industria musical: un entendimiento necesario}

El mercado discográfico español se caracteriza por un lado por su gran atomización internacional (Buquet, 2002; Calvi, 2006) y por otro por una gran presencia del mercado independiente (Albornoz y Gallego, 2012). Este acaparamiento por parte de las majors trae consigo una estandarización del repertorio, que se vuelve plenamente internacional como podemos observar en la composición del repertorio radiado del siguiente gráfico:

\section{nacional $\quad$ Internacional}

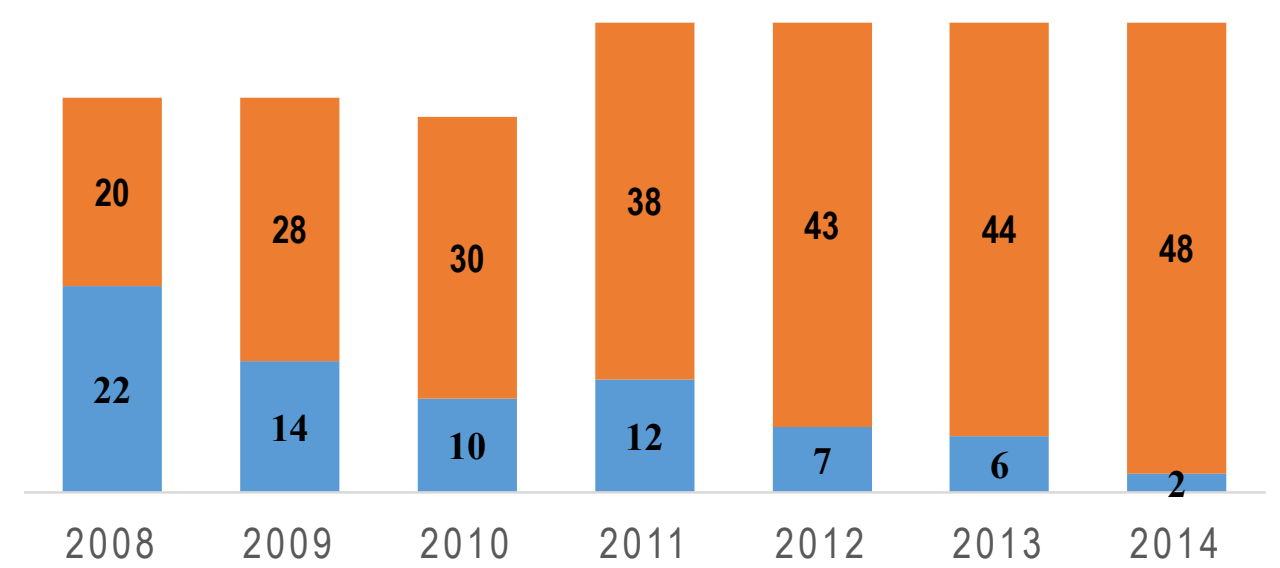

Gráfico 1. Número de artistas del repertorio nacional e internacional en las 50 canciones más radiadas en España (2008-2015). Fuente: SGAE $(2010,2011,2012,2013,2014 b, 2015$ y 2016).

La promoción de la música local y muy especialmente relacionado con las lenguas cooficiales está en el ADN de las emisoras musicales públicas de Catalunya y Euskadi. Mantener el equilibrio entre la presencia de las grandes multinacionales y la industria local no siempre es fácil. Así el director de Gaztea Irratia relata una situación en la que a las presiones de las grandes discográficas une el trato con los sellos locales que editan su música mayormente en euskera: «Yo entiendo que si hacemos una selección de lo que se produce es a favor del euskera, si lo pinchamos absolutamente todo es en contra del euskera. ¿Qué pasa con las discográficas? Que prefieren tener pocos oyentes pero de los suyos. Entonces normalmente las relaciones son un poco tensas en un principio, luego nos llevamos bien. Pero es que claro, todo el sector está en crisis», señala el director de Gaztea Irratia Jon Lamarca (2013).

La presencia de los sellos discográficos en la radio especializada musical española es un indicador de pluralismo o en este caso de su ausencia. De hecho en 2003 la UFI sugirió en su Libro Blanco una cuota obligatoria de música independiente en los medios de comunicación (UFI, 2003). Sin embargo, tal y como podemos ver en el siguiente gráfico la presencia de las majors es mayoritaria:

Gráfico 2. Sello discográfico del Top 50 de las canciones más radiadas (14/10/2016-20/10/2016)

Fuente: elaboración propia a partir de Promusicae (2016).

La mayoría de entrevistados señalan una relación fluida con la industria musical aunque siempre en términos de colaboración: «Nosotros somos el escaparate que ellos necesitan y ellos son el alimento que nosotros necesitamos. Estamos condenados a entendernos», señala la coordinadora de Canal Fiesta Radio, Araceli Limón (2015). Además, los entrevistados señalan como los acuerdos con «los actores musicales», en palabras de Tomás Fernando Flores de Radio 3, como son las salas de conciertos o festivales de música son fundamentales en la estrategia de las emisoras. En líneas generales podríamos decir que hay dos clasificaciones entre las emisoras estudiadas: aquellas centradas en la música más «independiente» (iCat.cat y Radio 3) y las que son más «comerciales» (Canal Fiesta Radio y Gaztea Radio). Así, los entrevistados de Radio 3 e iCat.cat señalan el gusto «no comercial» de las emisoras. En cambio, el director de Gaztea Irratia remarca el empleo de la música comercial como «elemento netamente relacionado con el entretenimiento más juvenil» (Lamarca, 2013) y empleado como una decisión directamente relacionada con la audiencia: «La gran crisis de Gaztea fue en el año 2006 y ahí se estudió que nos guste o no las minorías las dejas aparte y no tenemos presupuesto para todo» (ídem). Las relaciones con la industria más independiente son fundamentales para la los directores de iCat.cat y Radio 3 : «porque ellos saben que el principal escaparate que tienen, en algunos casos el único es Radio 3» (Tomás Fernando Flores, 2016). 
«Después de 50 años, Raphael se ha vuelto indie». Esta frase pronunciada por Ángel Carmona, conductor de Hoy empieza todo en Radio 3 , en una entrevista al artista español Raphael nos da un ejemplo de la complejidad y confusión que conllevan los conceptos indie/mainstream (Hesmondhalgh y Meier, 2015; Wikström, 2013). La industria discográfica independiente ha estado tradicionalmente asociada al descubrimiento de talento, mientras que las grandes multinacionales buscarían el beneficio económico. No obstante, el concepto «independiente» puede ser calificado de manera estética (Hesmondhalgh, 1997) como apunta el director de Radio 3, Tomás Fernando Flores (2014): «Es que el concepto independiente casi es un concepto estético. Los grandes grupos independientes españoles, como Los Planetas, son de una multinacional. Yo creo que independiente se refiere más bien al sentido de la propuesta artística que hacen». Por tanto podríamos hablar más bien de música de producción independiente más que de difusión independiente, lo que es una barrera más difícil de delimitar. La radio actúa no solo como promotor sino también como prescriptor de contenidos. Existe una serie de artistas llamados «emergentes» que en su mayoría están al margen de la industria musical. Así, Gaztea Irratia, iCat.cat y Radio 3 tienen concursos de maquetas para artistas emergentes llamados Maketa Lehiaketa, Sona9 y Proyecto Demo respectivamente. El objetivo de estos programas es «impulsar» la carrera musical de un artista 5 , aunque en ocasiones esta labor de descubrimiento no se ha traducido en un apoyo posterior cuando el artista ya forma parte del engranaje industrial, como remarca Lucía Flores (2014), coordinadora musical de iCat.cat: «Nosotros tenemos en ese sentido un mal hábito. Y es que hemos generado éxitos de grupos emergentes o nuevos y cuando se han convertidos en grandes éxitos los hemos abandonado». Para una emisora orientada a la música comercial como es Gaztea Irratia, el encaje programático de los músicos desconocidos resulta complicado. Su director señala la apuesta por dar una oportunidad pero a aquellos grupos que ya se han dado a conocer en cierta medida, a veces en su propio concurso de talentos, para lo que destaca el papel de la programadora musical en esta tarea: «A ver, es una relación difícil. Normalmente los grupos que están sonando en euskera o locales es porque ya han demostrado algo, o bien porque han ganado algún premio, Maketa Lehiaketa o bien porque tienen muchas visitas en Internet» (Lamarca, 2013). Por su parte, Canal Fiesta Radio, tenía un programa orientado a este fin, Estrénate en el Fiesta, que se dejó de emitir en 2013. A propósito de este último punto señalaba su directora la nueva orientación de la emisora: «Tenemos un proyecto en marcha que se llama Revelación Fiesta, que es apoyar durante un mes a un artista que ha grabado su primer disco o que está a punto de grabarlo. 0 sea, no se trata de un cazatalentos, se trata que una vez que una discográfica ha fichado a un artista, nosotros le apoyamos en sus momentos más difíciles, que son el principio. Lo que estamos apoyando es al artista y a la gente que ha apostado por él» (Limón, 2015).

\section{Conclusiones}

En el presente artículo hemos realizado una aproximación a la relación entre radio musical pública e industria musical. A priori, ésta no se ve influida por la orientación comercial o independiente de las emisoras estudiadas. Por un lado tenemos las emisoras con una música definida como independiente», como iCat.cat y Radio 3. Por otro, tenemos las radios consideradas comerciales», que es el ejemplo de Canal Fiesta Radio y Gaztea Irratia. En ambos casos los entrevistados manifestaron tener independencia con respecto a la industria musical, aunque en el caso de las emisoras comerciales esta relación es más estrecha a la par que complicada. En tiempos de crisis la radio pública debe apoyar a la industria musical, no solo la fonográfica sino también a otros actores como las salas de conciertos y los festivales de música.

Entre la radio y la industria se sitúan los artistas emergentes, con los cuales todos los entrevistados muestran un compromiso importante. La abundancia de contenidos que ofrece Internet empuja a la radio a encontrar una manera de seguir siendo atractiva e interesante. En este sentido el contacto con la industria musical más local es fundamental para alimentar el circuito y seleccionar en cierta medida artistas que independientemente de si serán un referente o no, siempre estarán ligados a «su radio».

Las líneas de investigación que este artículo ha intentado abrir deberían ampliarse con estudios dirigidos a la industria musical, muy especialmente la de carácter local e independiente, así como a los artistas emergentes. En definitiva, la prescripción de una buena selección musical es uno de los pilares de la radio musical y constituye una oportunidad única para actuar como enlace entre industria, radio y oyentes.

\section{Referencias bibliográficas}

Albornoz, L.A. \& Gallego, I. (2012). La industria de la música popular en España: los sellos independientes en la era digital. Revista da Associação Nacional dos Programas de Pós-Graduação em Comunicação E-compós, 15(2), 1-19.

Amoedo, A., Martínez-Costa, M. P. \& Moreno, E. (2008). An analysis of de communication strategies of Spanish comercial music networks on the web: los 40. com, los 40 principales.com, cadena100.es, europafm.es and kissfm.es. The Radio Journal: International Studies in Broadcast and Audio Media, 6(1), 5-20.

Arboledas, L. (2009). La industria radiofónica en Andalucía: dependencia económica y control político (1982-2008). Tesis doctoral: Granada, Universidad de Granada

Barnard, S. (1989). On the Radio. Music radio in Britain. Philadelphia: Open University Press.

Bonet, M., Fernández-Quijada, D. \& Ribes, X. (2011). The Changing Nature of Public Service Radio: A Case Study of iCat FM. Convergence: The International Journal of Research into New Media Technologies, 17(2), 177-192.

Buquet, G. (2002). La industria discográfica: reflejo tardío y dependencia del mercado internacional. En Bustamante, E. (ed). Comunicación y cultura en la era digital. Industrias, mercados y diversidad en España (pp. 67-101). Barcelona: Editorial Gedisa, S.A.

Caffarel, C. (2007). Hacia la Radio Televisión Española de los ciudadanos. Madrid: Ediciones Laberinto.

${ }^{5}$ A este propósito señalaba Xose Luís Freire, antiguo coordinador de RG Música, en una comunicación personal (2014) como el estudio de grabación de Radio Galega provocó en sus inicios algunos conflictos con la escasa industria musical gallega. 
Calvi, J. (2006). Plan integral de apoyo a la música y a la industria discográfica. Documento de trabajo Fundación Alternativas (http://www.falternativas.org/en/laboratorio/documentos/documentos-de-trabajo/plan-integralde-apoyo-a-la-musica-y-a-la-industria-discografica) $(01-07-2013)$

CCMA (Corporació Catalana de Mitjans Audiovisuals). (2009a). Comptes anuals consolidats i informe de gestió 2008 (http://www.ccma.cat/corporacio/corporacio_informe_anual_cat.htm) (28-01-2014)

CCMA (Corporació Catalana de Mitjans Audiovisuals). (2009b). Memòria anual d'activitats 2008 (http://www.ccma.cat/corporacio/corporacio_informe_anual_cat.htm) (28-01-2014)

CCMA (Corporació Catalana de Mitjans Audiovisuals). (2010a). Comptes anuals consolidats i informe de gestió 2009 (http://www.ccma.cat/corporacio/corporacio_informe_anual_cat.htm) (28-01-2014)

CCMA (Corporació Catalana de Mitjans Audiovisuals). (2011a). Comptes anuals consolidats i informe de gestió 2010 (http://www.ccma.cat/corporacio/corporacio_informe_anual_cat.htm) (28-01-2014)

CCMA (Corporació Catalana de Mitjans Audiovisuals). (2011b). Memòria anual d'activitats 2010 (http://www.ccma.cat/corporacio/corporacio_informe_anual_cat.htm) (28-01-2014)

CCMA (Corporació Catalana de Mitjans Audiovisuals). (2013a). Comptes anuals consolidats i informe de gestió 2012 (http://www.ccma.cat/corporacio/corporacio_informe_anual_cat.htm) (28-01-2014)

$\begin{array}{llllll}\text { CCMA (Corporació Catalana de Mitjans } & \text { Audiovisuals). } & \text { (2014b). } & \text { Pla } & \text { d'activitats } 2014\end{array}$ (http://www.ccma.cat/corporacio/corporacio_informe_anual_cat.htm) (28-01-2014)

CCRTV (Corporació Catalana de Ràdio i Televisió). (2007a). Comptes anuals 2006 (http://www.ccma.cat/corporacio/corporacio_informe_anual_cat.htm) (28-01-2014)

CCRTV (Corporació Catalana de Ràdio i Televisió). (2007b). Informe Anual 2006 (http://www.ccma.cat/corporacio/corporacio_informe_anual_cat.htm) (28-01-2014)

Congreso de los Diputados. (2005a). Control parlamentario RTVE. Diario Oficial del

Congreso de los Diputados, 25 de mayo de 2005, núm. 290.

Congreso de los Diputados. (2005b). Control parlamentario RTVE. Diario Oficial del Congreso de los Diputados, 28 de junio de 2005, núm. 630.

Congreso de los Diputados. (2005c). Control parlamentario RTVE. Diario Oficial del Congreso de los Diputados, 29 de junio de 2005, núm. 336.

Congreso de los Diputados. (2005d). Control parlamentario RTVE. Diario Oficial del Congreso de los Diputados, 16 de febrero de 2005, núm. 155.

Congreso de los Diputados. (2005e). Control parlamentario RTVE. Diario Oficial del Congreso de los Diputados, 7 de abril de 2005, núm. 181.

Consejería de Cultura; EITB (Euskal Irrati Telebista). (2012). Contrato Programa con el Ente Público de Derecho Privado "Radio Televisión VascaEuskal Irrati Telebista" para el periodo 2012 y su anexo de concreción de compromisos al mismo" (http://www.eitb.com/multimedia/corporativo/documentos/contrato-programa-2012.pdf) (25-01-2014)

Consejería de la Presidencia e Igualdad. (2012). Acuerdo de 28 de diciembre de 2012, del Consejo de Gobierno, por el que se aprueba el Contrato-Programa entre el Consejo de Gobierno de la Junta de Andalucía y la Agencia Pública Empresarial de la Radio y Televisión de Andalucía para el período 2013-2015 (http://www.juntadeandalucia.es/boja/2012/254/BOJA12-254-00019-21039-01_00019112.pdf) (30-04-2015)

Consejo para la Reforma de los Medios de Comunicación de Titularidad del Estado. (2005). Informe para la reforma de los medios de comunicación de titularidad del Estado (http://estaticos.elmundo.es/documentos/2005/02/21/reforma.pdf) (02-10-2013)

Crisell, A. (2006). More than a Music Box: Radio Cultures and Communities in a Multi-Media World. Londres: Berghahn Books.

Departamento de Presidencia; Consejería de Hacienda y Administración Pública; Consejería de Cultura; EITB (Euskal Irrati Telebista). (2007). Contrato-programa con el ente público Radio Televisión vasca (EITB) para el periodo 2007-2010 (http://www.kultura.ejgv.euskadi.net/r46kghz2/es/contenidos/informacion/eitb/es_eitb/adjuntos/EITB_0710.pdf) (24-01-2014)

EITB (Euskal Irrati Telebista). (2007). Compromisos de EITB con la sociedad: contrato social con la audiencia (http://www.parlamento.euskadi.net/irud/08/00/005153.pdf) (04-02-2014) 
EITB (Euskal Irrati Telebista). (2011a). Memoria EITB 2009-2010 http://www.eitb.com/multimedia/documentos/2011/07/22/515224/anexo_a_es.pdf) $(27-01-2014)$

EITB (Euskal Irrati Telebista). (2011b). Cuentas anuales consolidadas al 31 de diciembre de 2011 e informe de gestión del ejercicio 2011 (http://www.eitb.com/multimedia/documentos/2012/06/26/678418/auditoria_2011.pdf) (24-05-2014)

EITB (Euskal Irrati Telebista). (2012a). Normas reguladoras de la emisión de publicidad en los medios de difusión de ElTB (http://www.eitb.com/multimedia/corporativo/documentos/normas-reguladoras-de-la-emision-de-publicidaden-medios-difusion-eitb.pdf) (24-052014)

EITB (Euskal Irrati Telebista). (2012b). Plantilla Grupo EITB 2010-2011 (http://www.eitb.com/multimedia/corporativo/documentos/relacion-plantillasgrupo-EiTB.pdf) (24-05-2014)

EITB (Euskal Irrati Telebista). (2012c). Cuentas anuales consolidadas al 31 de diciembre de 2012 e informe de gestión del ejercicio 2012 (http://www.eitb.com/multimedia/corporativo/documentos/cuentas-anuales-auditadas-eitb-2012-consolidado.pdf) (24-05-2014)

EITB (Euskal Irrati Telebista). (2012d). Albiste testu bikainak (libro de estilo) (http://www.eitb.com/multimedia/corporativo/documentos/albiste-testubikainak-euskaraz-eitb.pdf) (24-05-2014)

EITB (Euskal Irrati Telebista). (2012e). Estatuto de redacción de la Radio Televisión Vasca-Euskal Irrati Telebista y sus sociedades filiales (http://www.eitb.eus/multimedia/corporativo/documentos/estatuto-de-redaccion-radiotelevision-vasca-y-sociedades-filiales.pdf) (24-05-2014)

EITB (Euskal Irrati Telebista). (2013a). Memoria EITB 2011-2012 (http://www.eitb.com/es/grupo-eitb/eitbplus/responsabilidad-social-corporativa) (24-05-2014)

Fouce, H. (2008). Emociones en lugar de soluciones. Música popular, intelectuales y cambio político en la España de la Transición. TRANSRevista Transcultural de música, 12 (http://www.sibetrans.com/trans/trans12/art20.htm) (25-02-2011)

Frith, S. (1987). Art into pop. Londres: Methuen.

Gallego, J.I. (2015). User-Generated Playlists: Radio Music Programming in the Age of Peer-to-Peer Production, Distribution, and Consumption. En Bonini, T. \& Monclús, B. (eds). Radio Audiences and Participation in the Age of Network Society (pp. 195-211). Londres: Routledge.

Gilbert, J.; Pearson, E. (2003). Cultura y políticas de la música dance. Disco, hip-hop, house, techno, drum'n'bass y garaje. Barcelona: Paidós Comunicación.

Gutièrrez, M., Ribes, X. \& Monclús, B. (2011). La audiencia juvenil y el acceso a la radio musical. Comunicación y Sociedad, XXIV(2), 305-331.

Hendy, D. (2000a). A Political Economy of Radio in the Digital Age. Journal of Radio \& Audio Media, 1(7), 213-234.

Hendy, D. (2000b). Pop music radio in the public service: BBC Radio 1 and new music in the 1990s. Media, Culture \& Society, $22,743-761$.

Hennion, A. \& Meadel, C. (1986). Programming music: radio as mediator. Cultural Society, 1(1), 97-114.

Hesmondhalgh, D. (1997). Post-Punk's Attempt to Democratise the Music Industry: The Success and Failure of Rough Trade. Popular Music, 16(3), 255-274.

Hesmondhalgh, D. \& Meier, L.M. (2015). Popular music, independence and the concept of the alternative in contemporary capitalism. En Bennett, J. \& Strange, N. (eds). Media Independence: Working with Freedom Or Working for Free? (pp. 94-116). New York: Routledge.

IFPI (International Federation of the Phonographic Industry). (2010b). Investing in music. How music companies discover, develop \& promote talent (http://www.ifpi.org/content/library/investing_in_music_2010.pdf) (03-07-2013)

Long, P. 2006. The primary code: The meanings of John Peel, radio and popular Music. The Radio Journal - International Studies in Broadcast and Audio Media, 4 (1,2,3), 25-48.

Martí, J.M. (1990). Modelos de programación radiofónica. Barcelona: Feed-Back Ediciones.

Moreno, E. (1999a). La música en la radio: transformación de un contenido en un concepto de programación. Tesis doctoral: Pamplona, Universidad de Navarra. 
Parlament de Catalunya. (1998). Llei 1/1998, de 7 de gener, de Política Llingüística (http://noticias.juridicas.com/base_datos/CCAA/ca-I11998.html) (22-01-2014)

Parlamento de Andalucía. (2010). Comisión de Control de la Agencia Pública Empresarial de la RTVA y de sus sociedades filiales. Diario de sesiones, 23 de febrero de 2010, núm. 306.

Pedrero Esteban, L. M. (2000). La radio musical en España. Historia y análisis. Madrid: IORTV.

Percival, M.J. (2011). Music Radio and the Record Industry: Songs, Sounds, and Power. Popular Music and Society, 34(4), $455-473$.

Percival, M.J. (2012). United Kingdom Music Radio Programming: Good Radio Records and the Imagined Audience. En Mollgaard, M. (ed). Radio and society: New thinking for an old medium (pp. 115-133). Cambridge: Cambridge Scholars Publishing.

Prado, E. (1995). El nuevo sistema radiofónico y la configuración del Estado: la sintonía de la radio española. En Díaz-Nosty, B. (dtor). Comunicación social 1994/Tendencias (pp. 235-240). Madrid: Fundesco.

Promusicae. (2016), TOP 50 - Lista de Radio Musical Semana 42: Del 15-10-2016 al 21-10-2016 (http://www.promusicae.es/listas/semanales?page=3) (30-10-2016)

Rothenbuhler, E. W. (2004). Rock and Roll Format. Museum of Broadcast Communications Encyclopedia of Radio, 3, $1219-1221$.

Rothenbuhler, E. W. \& McCourt, T. (1987) Commercial Radio and Popular Music. Processes of Selection and Factors of Influence. En Lull, J. (ed). Popular Music and Communication (pp. 101-115). Londres: Sage.

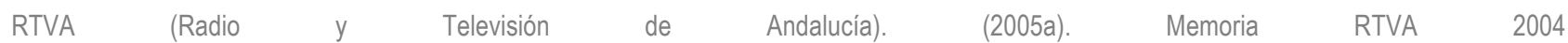

(http://www.canalsur.es/html/portal/com/bin/contenidos/zona_descarga/memoria_2004/1185531536322_rtva_memoria_2004.pdf) (25-01-2014)

RTVA (Radio y Televisión de Andalucía). (2005b). Memoria correspondiente al ejercicio anual terminado el 31 de diciembre de 2004 (http://www.juntadeandalucia.es/haciendayadministracionpublica/servicios/fiscalizacion/rendicion/cuenta2004/descargas/empresas/rtva_cons.pdf) $(17-05-2014)$

RTVA (Radio y Televisión de Andalucía). (2005c). Cuenta de resultados y balance Canal Sur Radio 2004 (http://www.juntadeandalucia.es/haciendayadministracionpublica/servicios/fiscalizacion/rendicion/cuenta2004/empresas.htm) (17-05-2014)

RTVA (Radio y $\quad$ Televisión de Andalucía). (2006a). Memoria $\quad$ RTVA 2005 (http://www.canalsur.es/html/portal/com/bin/contenidos/zona_descarga/memoria_2005/1156756614422_rtva_memoria_2005.pdf) (17-05-2014)

RTVA (Radio y Televisión de Andalucía). (2006b). Memoria correspondiente al ejercicio anual terminado el 31 de diciembre de 2005 (http://www.juntadeandalucia.es/haciendayadministracionpublica/servicios/fiscalizacion/rendicion/cuenta2005/descargas/empresas/cuentas/rtva_co ns.pdf) (17-05-2014)

RTVA (Radio y Televisión de Andalucía). (2006c). Cuenta de resultados y balance Canal Sur Radio 2005 (http://www.juntadeandalucia.es/haciendayadministracionpublica/servicios/fiscalizacion/rendicion/cuenta2005/empresas.htm) (17-05-2014)

RTVA (Radio y Televisión de Andalucía). (2006d). Defensor de la audiencia. Informe 18 abril (http://blogs.canalsur.es/defensorrtva/2006/04/27/informe-18-abril) (17-05-2014)

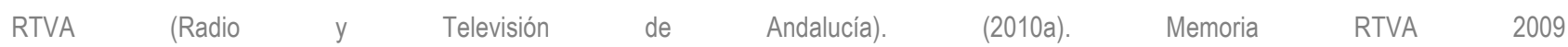
(http://www.canalsur.es/resources/archivos/2011/12/2/1322828886969RTVA_2010_alta.pdf) (17-05-2014)

RTVA (Radio y Televisión de Andalucía). (2010b). Memoria correspondiente al ejercicio anual terminado el 31 de diciembre de 2009 (http://www.juntadeandalucia.es/haciendayadministracionpublica/servicios/fiscalizacion/rendicion/cuenta2010/agencias.htm) (17-05-2014)

RTVA (Radio y Televisión de Andalucía). (2010c). Cuenta de resultados y balance Canal Sur Radio 2009 (http://www.juntadeandalucia.es/haciendayadministracionpublica/servicios/fiscalizacion/rendicion/cuenta2009/sociedades_i.htm) (17-05-2014)

RTVE (Radio Televisión Española). (2009a). Oficina de la defensora del espectador, oyente y usuario de medios interactivos. Tercer Informe Trimestral Enero, Febrero y Marzo de 2009 (http://www.rtve.es/rtve-responde/informes) (17-05-2014)

RTVE (Radio Televisión Española). (2009b). Oficina de la defensora del espectador, oyente y usuario de medios interactivos. Cuarto Informe Trimestral Abril, Mayo y Junio de 2009 (http://www.rtve.es/rtve-responde/informes) (17-05-2014) 
RTVE (Radio Televisión Española). (2009c). Oficina de la defensora del espectador, oyente y usuario de medios interactivos. Cuarto Informe Trimestral Julio, Agosto y Septiembre de 2009 (http://www.rtve.es/rtve-responde/informes) (17-05-2014)

RTVE (Radio Televisión Española). (2009d). Oficina de la defensora del espectador, oyente y usuario de medios interactivos. Informe Trimestral Octubre, Noviembre, Diciembre 2009 (http://www.rtve.es/rtve-responde/informes) (16-04-2015)

RTVE (Radio Televisión Española). (2009e). Memoria 2008 sobre el cumplimiento de la función de servicio público (http://www.rtve.es/rtve/20140609/transparencia-memorias-servicio-publico/951181.shtml) (16-04-2015)

RTVE (Radio Televisión Española). (2010a). Oficina de la defensora del espectador, oyente e internauta. Informe trimestral Abril, Mayo, Junio 2010 (http://www.rtve.es/rtve-responde/informes) (16-04-2015)

RTVE (Radio Televisión Española). (2010b). Oficina de la defensora del espectador, oyente e internauta. Informe Trimestral Julio, Agosto y Septiembre de 2010 (http://www.rtve.es/rtve-responde/informes) (consulta 16/04/2015)

RTVE (Radio Televisión Española). (2010c). Oficina de la defensora del espectador, oyente e internauta. Informe trimestral Octubre, Noviembre, Diciembre 2010 (http://www.rtve.es/rtve-responde/informes) (consulta 16/04/2015)

RTVE (Radio Televisión Española). (2010d). Memoria 2009 sobre el cumplimiento de la función de servicio público (http://www.rtve.es/rtve/20140609/transparencia-memorias-servicio-publico/951181.shtml) (consulta 16/04/2015)

RTVE (Radio Televisión Española). (2010e). Manual de Estilo de RTVE. Directrices para los profesionales (http://manualdeestilo.rtve.es) (16-042015)

RTVE (Radio Televisión Española). (2013d). Oficina de la defensora del espectador, oyente e internauta. Informe sobre el cumplimiento del Código de Protección de la Infancia y la Juventud (http://www.rtve.es/rtveresponde/informes) (16-04-2015)

RTVE (Radio Televisión Española). (2013e). Oficina de la defensora del espectador, oyente e internauta. Informe trimestral sobre el cumplimiento del Código de Protección de la Infancia y la Juventud. Enero, Febrero y Marzo 2013 (http://www.rtve.es/rtve-responde/informes) (16-04-2015)

RTVE (Radio Televisión Española). (2013f). Oficina de la defensora del espectador, oyente e internauta. Informe trimestral sobre el cumplimiento del Código de Protección de la Infancia y la Juventud. Julio, Agosto y Septiembre 2013 (http://www.rtve.es/rtve-responde/informes) (16-04-2015)

RTVE (Radio Televisión Española). (2013g). Memoria 2012 sobre el cumplimiento de la función de servicio público y la responsabilidad social corporativa (http://www.rtve.es/rtve/20140609/transparencia-memorias serviciopublico/951181.shtml) (16-04-2015)

RTVE (Radio Televisión Española). (2013h). Programación responsable 2013 (http://www.rtve.es/rtve/20140526/rc-programas/943300.shtml) (consulta 16/04/2015)

Stiernstedt, F. (2014). The Political Economy of the Radio Personality. Journal of Radio \& Audio Media, 21(2), 290-306.

UFI (Unión Fonográfica Independiente). (2003). Libro blanco (http://ufimusica.com/new/wpcontent/uploads/Libro-Blanco-de-UFi.pdf) (06-05-2014)

Wall, T. \& Dubber, A. (2009a). Specialist music fans online: implications for public service broadcasting. Listener online engagement with BBC Radio programming (http://www.bbc.co.uk/blogs/legacy/knowledgeexchange/birmingham.pdf) (20-11-2014)

Wall, T.; Dubber, A. (2009b). Specialist music, public service and the BBC in the Internet age. The Radio Journal-International Studies in Broadcast and Audio Media, 7(1), 27-47.

Wikström, P. (2013). The Music industry: music in the cloud. Cambridge: Polity Press. 


\section{Cómo citar este artículo en bibliografías - How to cite this article in bibliographies / references:}

COSTA GÁLVEZ, L. (2017): “¿Indie vs. mainstream? Una aproximación a la relación entre industria musical y radio musical pública en España”. En Revista de la Asociación Española de Investigación de la Comunicación, vol. 4, número 8, pp. 63-72. 